\title{
Repurposing Case-Based Learning to a Conversational Agent for Healthcare Cybersecurity
}

\author{
Matthew PEARS ${ }^{\text {, }}$, James HENDERSON ${ }^{\mathrm{a}}$ and Stathis Th. KONSTANTINIDIS ${ }^{\mathrm{a}, 1}$ \\ ${ }^{\text {a }}$ School of Health Sciences, University of Nottingham, UK
}

\begin{abstract}
A crucial factor for successful cybersecurity education is how information is communicated to learners. Case-based learning of common cybersecurity issues has been shown to improve human behaviour for prevention. However, some delivery methods prevent realistic critical appraisal and reflection of awareness. Conversational agents can scaffold healthcare workers' understanding and promote deterrence strategies. The challenges of repurposing material to create a case-based agent were explored, and the ASPIRE process was modified. Heuristic evaluation from 10 experts in innovative educational technology resulted in the desired outcomes of usability, however Natural Language Understanding improvements were needed. Discussion of best practice when repurposing into conversational agents suggested modification of the ASPIRE process is feasible for future use.
\end{abstract}

Keywords. Cybersecurity, Chatbot, Dialogue, RASA, Social Media, Healthcare workforce IT skills, Information Security

\section{Introduction}

The current healthcare spotlight is on human-computer vulnerability in cybersecurity, being the most successful in attacks to data [1]. A persistent challenge in cybersecurity is how to help individuals acquire skills that enable them to adapt to the dynamic landscape of threats. Threats online both in and out of work, and in a variety of presentation methods, such as emails or social media requests, can increase vulnerability [2]. It is the non-technical skills of a healthcare worker that are the mediators to how well knowledge is used to adapt behaviour [3] and current research is now focusing on the human components when using social media and other communication platforms. The need for improved non-technical skills in cybersecurity has not been met despite emphasis been placed on such risks at least a decade ago [4]).

There are few materials that provide a scaffolding approach to improve nontechnical skills. Scaffolding of cognition when learning is the individualized support from an educator who aids in a reciprocating process of presenting appropriate and timely information. Case-based learning is one method to evoke cognitive scaffolding as accurate and robust cognitive models of good/bad cybersecurity behaviours and threats are learned through inquisitive interaction with realistic cases. However, most current

\footnotetext{
${ }^{1}$ Corresponding Author: Dr Stathis Th Konstantinidis, School of Health Sciences, University of Nottingham, B floor, QMC, Nottingham, NG7 2HA, UK; E-mail: Stathis.Konstantinidis@nottingham.ac.uk.
} 
training delivery methods present the learner with a reduced and predetermined number of decisions. This impedes critical appraisal and decision-making abilities when the learner is attempting to be independent in real-life prevention strategies. One solution is the use of chatbots- they can scaffold a learner's understanding of concepts, resulting in increased probability of being transferred into similar real-world scenarios $[5,6]$. Scaffolding-based chatbots can better create meaningful interactions with a learner compared to non-scaffolding structures and results in improved information retention [7]. Therefore, a first-person case-based, reusable AI chatbots has theoretical grounds to be an effective educational tool.

The aims of the current work are: to modify the ASPIRE [8] model towards chatbot design. Obstacles encountered in this process required creative problem solving to transform the information, structure, and interface into the final product design. To subsequently repurpose a previous case-based material that has content shown to be of poor/unsecure behaviours in healthcare workers [9]. Repurposing existing high-quality content reduces the development costs and ensures accuracy of the context. To complete the ASPIRE model by heuristic evaluation of the agent to determent if an easy to use and beneficial agent can be established.

\section{Method}

Participants: Ten staff members within the Health E-Learning Media (HELM) team at the University of Nottingham were recruited. They consisted of learning technologists, software research developers, digital/e-learning academics, and other research professionals in e-learning and media.

Design: The creation of the agent was guided by user-centred design as based on the content of a previous case-based material. Yet as chatbots differ from typical educational online materials, the co-creation methodology of the 'ASPIRE' model (Aims, Storyboarding, Population, Implementation, Release, and Evaluation) framework was adapted for chatbot development following the ERASMUS+ CEPEH project [10].

Materials: Being customized for chatbots, the Chatbot Usability Questionnaire (CUQ) was used alongside the prevalent System Usability Scale Score (SUS) [11]. Multiple metrics are more appropriate when measuring usability of chatbots [12] therefore a combination of two scores can provide an all-inclusive overview. There were 26 questions overall.

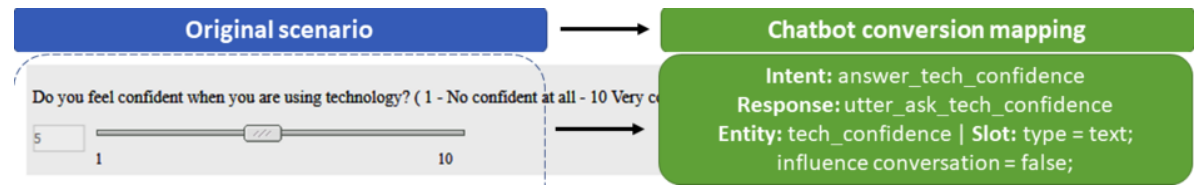

Figure 1: Mapping example of the CBL scenario to Chatbot

Procedure: A specification of the chatbot was created from an existing case-based learning scenario, which included desired response paths and social characteristics of the chatbot. Modified development steps are depicted in Table 2. While the Path in the chatbot follows the scenario's flow, the text from the different case nodes had to be analysed and hand create "Intends" to establish the purpose behind the different statements and "Entities" to classify the purpose of the users behind every statement (Figure 1). Then implemented into RASA [13], and Natural Language Understanding 
(NLU) training followed, allowed users partial circumventive discussion, however the structure would deterministically continue with the desired training material. Persuasive techniques [14]. were used to lead the learner to follow a conversation path enhancing the learning experience and still feeling natural in conversation. Participants were recruited via University internal email.

\section{Results}

Participants either strongly or moderately agreed that: the agent was not complex $(100 \%)$, technical support is not required $(100 \%)$, the agent was welcoming and friendly $(100 \%)$, made the users feel interested $(100 \%)$, the agent was easy to use $(90 \%)$, users felt confident during use (90\%), and the agent appeared transparent/clear $(90 \%)$. Feedback of suboptimal elements suggested that the agent was too robotic $(60 \%)$ along with limitations in presenting a realistic personality $(50 \%)$, this may have been a factor for $60 \%$ agreeing $/ 40 \%$ neutral in that they would like to increase the Frequency of Use of the agent.

To investigate influencing factors in Frequency of Use (being to use the chatbot again), a Kendall Correlation analysis was performed. Strength and direction of the 25 questions from the SUS and CUQ questions highlighted significant correlations. Frequency of use, quick to learn/learn before use, and confidence during use has significant positive correlations between each other (see Table 1), all with large effect sizes. This met the criteria in the aims in being simple/natural to use.

Being too robotic and not showing sufficient realism in personality, were not significantly negatively correlated with Frequency of Use. This meant they each had a weak to moderate influence, as did other measures. This can be addressed with further development. Thematic analysis of open-ended feedback suggested limited input ranges caused repetition of certain sections, this can also be addressed with further development and may explain the source of lower ratings for being robotic and reduced realism of personality.

Table 1. Significant correlations from the users' experience on frequency of using the agent in the future

\begin{tabular}{lccl}
\hline \multicolumn{1}{c}{ Combination } & $\mathbf{r}_{\mathbf{k}}$ & $\boldsymbol{p}$ & \multicolumn{1}{c}{ Frequency of Use will increase: } \\
\hline Frequency of Use* Quick to learn & 0.69 & 0.027 & -as learning speed increases \\
Frequency of Use* Confidence & 0.64 & 0.045 & -as confidence increases \\
Frequency of Use* Learn Before Use & -0.69 & 0.026 & -as learning before use decreases \\
\hline
\end{tabular}

\section{Discussion and Conclusions}

The aim of modifying the ASPIRE process was met, as the Storyboard stage was changed from previous collaborative visual idea generation to decision-making tree, and content driven discussion. The aim of repurposing a previous case-based material was met we effectively recreated the data into relevant RASA-based intents, slots, stories, NLU etc. (see below). The aim to evaluate the agent showed us that the SUS and CUQ questions complemented each other in suggesting the agent had simplistic usability, well-defined information, but limited range of conversational flow, due to being a trail version. This resulted in some users' perceptions of personality being broken/reduced.

There are many considerations as to how material can be reused for chatbot agent development to promote an inquisitive approach in a real-time role-playing environment. 
Table 2 depicts an overview of conversational agent adapting processes, describing the challenges and the solutions we identified following the ASPIRE framework.

Table 2. Challenges and Solutions utilizing ASPIRE framework to repurpose CBL to a Conversational Agent.

\begin{tabular}{|c|c|c|}
\hline ASPIRE & Procedure and Challenges & Tips and Solution \\
\hline Aim & Aim remained the same & $\begin{array}{l}\text { Learner should receive clearly information } \\
\text { around the background, aims or objectives }\end{array}$ \\
\hline Storyboarding & $\begin{array}{l}\text { - OpenLabyrinth tree structure graph } \\
\text { converted to a graph including } \\
\text { entities and intends } \\
\text { - The intended paths were relatively } \\
\text { easy to replicate into a chatbot }\end{array}$ & $\begin{array}{l}\text { New stakeholders were not required as } \\
\text { specifications already available. }\end{array}$ \\
\hline Purpose & $\begin{array}{l}\text { - Specifications altered in order the } \\
\text { learner do not observe the dialogue, } \\
\text { but to be part of it } \\
\text { (observer -> role playing). } \\
\text { - Peer-review of the actual text is more } \\
\text { challenging as different paths and } \\
\text { alternatives answers can be given }\end{array}$ & $\begin{array}{l}\text { - Pervasive techniques embedded into the } \\
\text { dialogue in order to enable the learner to } \\
\text { follow the ideal path } \\
\text { - Language reviewed to ensure user-friendliness } \\
\text { and being non-robotic } \\
\text { - Reconsider the amount of text present in any } \\
\text { given message and be aware of the context, } \\
\text { tense and conversational tone } \\
\text { - Alternatives answers should be provided in the } \\
\text { "training" of the chatbot by experts }\end{array}$ \\
\hline Implementation & $\begin{array}{l}\text { - Multiple interactions at each point } \\
\text { are possible. } \\
\text { - Focus was on programmable logic, } \\
\text { and stories or rules to mimic desired } \\
\text { behaviours. } \\
\text { - Prevent the learner getting frustrated } \\
\text { by a poor learning experience } \\
\text { - 'Training' data provided by the } \\
\text { developer }\end{array}$ & $\begin{array}{l}\text { - Persuasive techniques used to keep learner on } \\
\text { track, error handling used for non-manageable } \\
\text { responses from the learner. } \\
\text { - 'training' data need multiple users in order to } \\
\text { be efficient and valid. } \\
\text { - set the parameters or responses which can pull } \\
\text { a learner back in by adding clarification or } \\
\text { remind of 'polite manners' to gently aid and } \\
\text { encourage }\end{array}$ \\
\hline Release & $\begin{array}{l}\text { The more "wise" (complicated) the } \\
\text { chatbot the more time needed to } \\
\text { recompile after small changes }\end{array}$ & $\begin{array}{l}\text { Emphasis should be given the design part of the } \\
\text { development and the early training of data. }\end{array}$ \\
\hline Evaluation & $\begin{array}{l}\text { Measure usability of chatbot including } \\
\text { chatbot personality, user experience } \\
\text { and error handling }\end{array}$ & $\begin{array}{l}\text { An iterative process should be followed as part } \\
\text { of an agile participatory approach }\end{array}$ \\
\hline
\end{tabular}

Chatbot characteristics identified are consistent with the existing literature (14), but still remain an open research field for educational chatbots. The unlimited input of the user in conversation with the chatbot is challenging to manage $100 \mathrm{~s}$ of potential responses in a logical and linguistic format that not only kept the user on the optimal learning path but achieved this with a positive effect on each user. Persuasive techniques are useful to keep the learner focused on the learning objectives. Once underpinning pedagogy and learning journey has been identified, the focus of development with traditional online learning is around the interface. An agile iterative approach following ASPIRE framework has been found useful in the development of the current chatbot and also similar methodologies have been proposed at a theoretical level [15]. Different types of conversational agents have been identified following blooms taxonomy $[16,17]$, however the challenges of co-creating them are open a lot and the created chatbot is listed in the higher level of bloom taxonomy as it triggers the learner to argue, defend and select her interactions.

Conversational agent creation via repurposing of differently constructed material required support from the ASPIRE process and creativity from the software developer. 
However, participants' feedback was positive, and the concept of 1st person dialogue (role-playing) worked well. The evaluation provided the context and direction for subsequent development and testing with a large cohort of healthcare staff- for example in a single-blind, matched-pairs assessment with comparison against existing training materials. Despite the current limitation of small number of participants in the usability evaluation and the challenges in the development of the ASPIRE process, overall, the indications were positive for the continuing development of this novel conversational agent in cybersecurity training for the healthcare workforce.

\section{Acknowledgments}

This work is supported by the ERASMUS+ Strategic Partnerships for Vocational Education and Training "Training Blueprint for the Digital Transformation of Health and Care (TBDTHC)" (2018-1-IT01-KA202-006735) and the ERASMUS+ Strategic Partnership in Higher Education "Chatbot Enhance Personalise European Healthcare Curricula (CEPEH)" (2019-1-UK01-KA203-062091) projects of the European Union.

\section{References}

[1] Selvam VSD. Human Error in IT Security. arXiv preprint arXiv:200504163; 2020.

[2] Aldawood H, Skinner G. Educating and Raising Awareness on Cyber Security Social Engineering: A Literature Review. In Proc. 2018 IEEE TALE; 2018. p. 62-8.

[3] Data Security Awareness Workbook 2018. National Health Service: Airedale - trust. Available at: http://www.airedale-trust.nhs.uk/wp/wp-content/uploads/2019/01/Data-Security-AwarenessWorkbook.pdf. Accessed 2021 Jan 22.

[4] Forte D, Power R. The ultimate cybersecurity checklist for your workforce. Computer Fraud \& Security. 2007 Sep 1;(9):14-9.

[5] Applebee AN, Langer JA. Instructional scaffolding: Reading and writing as natural language activities. Language arts. 1983;60(2):168-75.

[6] Dolianiti F, Tsoupouroglou I, Antoniou P, et al. Chatbots in Healthcare Curricula: The Case of a Conversational Virtual Patient. In Proc. BFAL2020, LNCS, vol.12462. Springer,Cham.; 2020. p.137-47.

[7] Winkler R, Hobert S, Salovaara A, Söllner M, Leimeister JMS, the lecturer: Improving learning in online education with a scaffolding-based conversational agent. In Proc CHI 2020. 2020; p. 1-14.

[8] Windle R, Wharrad H, et al. Collaborate to create; Stakholder participation in Open content Creation'. In: Presented at Association for Learning Technology Conference (ALT-C). Warick; 2016.

[9] Coventry L, Branley-Bell D, Sillence E, et al. Cyber-Risk in Healthcare: Exploring Facilitators and Barriers to Secure Behaviour. In: Moallem A, editor. HCI for Cybersecurity, Privacy and Trust. Cham: Springer International Publishing; 2020. p. 105-22. (Lecture Notes in Computer Science).

[10] Pears M., et al. Co-Creation of Chatbots as an Educational Resource-Training The Trainers Workshop. In Proc of INTED 2021, (8-9 March), Valencia, Spain; 2021.

[11] Brooke J. SUS: a “quick and dirty'usability. Usability evaluation in industry. 1996;189.

[12] Kocabalil AB, Laranjo L, Coiera E. Measuring user experience in conversational interfaces: a comparison of six questionnaires. In 2018. p. 1-12.

[13] Nguyen T, Shcherbakov M. Enhancing Rasa NLU model for Vietnamese chatbot. International Journal of Open Information Technologies. 9(1):31-6.

[14] Bamidis PD, Konstantinidis ST, et al. Federating learning management systems for medical education: A persuasive technologies perspective. In Proc. of IEEE CBMS 2011; Bristol, UK 2011. p. 1-6.

[15] Bahja M, Hammad R, Butt G. A user-centric framework for educational chatbots design and development. In Springer; 2020. p. 32-43.

[16] Chaves AP, Gerosa MA. How Should My Chatbot Interact? A Survey on Social Characteristics in Human-Chatbot Interaction Design. International Journal of Human-Computer Interaction. 2020;1-30.

[17] Corral J. Artificially intelligent chatbots for health professions education. In: Konstantinidis S. et al. (eds) Digital Innovations in Healthcare Education and Training. Elsevier; 2020. p. 127-35. 